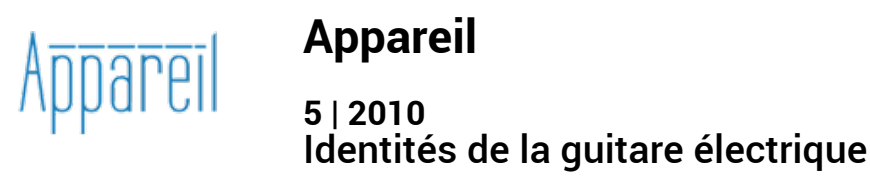

\title{
Résistance matérielle
}

Entretien avec Hasse Poulsen

\section{Otso Lähdeoja}

\section{(2) OpenEdition}

Journals

Édition électronique

URL : http://journals.openedition.org/appareil/1015

DOI : 10.4000/appareil. 1015

ISSN : 2101-0714

Éditeur

MSH Paris Nord

Référence électronique

Otso Lähdeoja, « Résistance matérielle », Appareil [En ligne], 5 | 2010, mis en ligne le 09 juin 2010, consulté le 30 juillet 2020. URL : http://journals.openedition.org/appareil/1015 ; DOI : https://doi.org/ 10.4000/appareil.1015

Ce document a été généré automatiquement le 30 juillet 2020

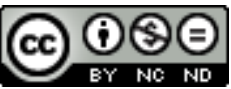

Appareil est mis à disposition selon les termes de la Licence Creative Commons Attribution - Pas d'Utilisation Commerciale - Pas de Modification 4.0 International. 


\title{
Résistance matérielle
}

\author{
Entretien avec Hasse Poulsen
}

\author{
Otso Lähdeoja
}

Je suis Hasse Poulsen, je suis guitariste. On peut dire que je joue du jazz... du jazz contemporain. Une musique basée sur l'improvisation, je suis aussi compositeur.

Comment approches-tu la guitare?

Hasse Poulsen : Je joue une guitare qui est à la fois acoustique et électrique, une guitare acoustique amplifiée. Précisément c'est une Guild jumbo F-50, achetée en 1991. Quand j'avais 22 ans, je faisais partie d'un groupe de salsa, il y avait un autre guitariste qui jouait de la guitare flamenco. Je jouais une Fender Telecaster. La physicalité et le son de la guitare acoustique m'ont tout de suite séduit. Pendant quelques années j'ai un peu tourné autour du pot, puis en 1991 j'ai essayé tout ce que je pouvais trouver comme guitares acoustiques à Copenhague, avant de tomber sur celle-ci. Un accord m'a suffit pour savoir que c'était la bonne. Après il m'a fallu dix ans pour apprendre à la jouer ! C'est une guitare lourde à jouer, physique.

Au début j'étais influencé par Mike Stern, je cherchais son jeu assez léger et fort à la fois, mais on ne peut pas produire ça sur une guitare acoustique, il y a d'autres règles, l'on doit jouer avec le poids et le côté physique.

Puis assez vite j'ai été fasciné par Derek Bailey. Il avait un son très attaqué, dur, et j'ai cherché à faire sortir les notes un peu de la même manière. J'ai eu une période très « musique improvisée » où j'utilisais constamment des préparations et des effets sur la guitare. Cela a duré cinq ou six ans.

En arrivant en France j'ai commencé à jouer dans le milieu du jazz français, au début comme «l'improvisateur» pendant que les autres jouaient des choses écrites. Puis j'ai pris goût à jouer des mélodies, des solos, de jouer plus ou moins traditionnellement avec des «beaux» sons comme Bill Frisell ou Pat Metheny... ou n'importe quel guitariste de jazz. 
Je me suis rendu compte que c'est toujours un travail, c'est toujours dur, difficile, c'est toujours une lutte : est-ce que je vais arriver à jouer ces notes, à tenir le tempo... Cela s'entend dans le jeu, ça fait partie de mon son.

Peux-tu parler de ton utilisation de la guitare préparée, du geste élargi ?

Hasse Poulsen : Les préparations font désormais partie de mon vocabulaire intime, je n'y pense plus particulièrement, c'est quelque chose d'intégré.

Les préparations peuvent être utilisées de manières différentes. On peut chercher à ressembler à d'autres instruments : utiliser des balais pour des sons percussifs, un archet pour des sons continus un peu comme la vièle à roue. On peut aussi aller vers des sons extramusicaux, bruitistes. C'est alors plus une question de geste, de performance, du visuel presque. La guitare devient autre chose, comme quand je joue d'un ressort attaché à la guitare. Le son exact produit a moins d'importance que le geste effectué. Cela a un côté spectaculaire auquel je suis sensible et attaché. Les derniers mois j'ai expérimenté avec deux ressorts avec lesquels je peux me déplacer à plusieurs mètres de la guitare et toujours jouer! Quand on joue de cette manière-là, cela devient une découverte : qu'est-ce qui va se passer si je fais ça ? J'aime retrouver cet état d'esprit quand je joue dans des contextes plus traditionnels : si les autres font ceci, qu'est-ce qui va se passer si je fais cela, comment les autres vont-ils réagir? De cette manière il est possible de se retrouver dans une sorte de monde sonore qu'on explore ensemble, même avec le public.

C'est intéressant d'avoir les deux côtés sur un même instrument: une technique très travaillée et maîtrisée mais aussi un domaine où l'instrument devient un objet inconnu, surprenant.

Hasse Poulsen : Oui, pour moi il y a beaucoup de manières et de niveaux de faire la musique, et je n'ai pas trouvé la bonne. J'aime bien chercher entre les différents niveaux, et j'aime aussi que des gens qui viennent de différentes traditions se rencontrent et jouent ensemble.

Pendant longtemps, j'avais des sortes de visions ou de rêves - et je pense que tout le monde qui travaille avec des préparations en a: on voit un objet et on se demande quel son ça pourrait faire avec la guitare. Cette démarche c'est comme s'approprier le monde environnant sur la guitare. Maintenant, avec l'expérience, cela s'est beaucoup réduit, je connais bien les résultats sonores de différents matériaux.

Actuellement la guitare s'étend vers la musique électronique, comment te positionnes-tu par rapport à cette extension?

Hasse Poulsen : Avec l'électronique et les préparations, il y a un moment où l'on se rend compte qu'on va ailleurs. Comme quand on joue avec des objets frappés sur la guitare : l'on se rapproche alors du percussionniste. Et puis d'un coup on se rend compte qu'on pourrait faire les mêmes sons avec complètement un autre instrument : une table en bois, une scie, une boîte de métal, plein de trucs et là c'est trop pour moi : pas artistiquement, j'apprécie beaucoup les autres qui font cela, mais personnellement je préfère retourner dans le monde de la guitare, rester guitariste.

C'est pareil avec les effets électroniques. Une saturation peut chauffer le son, donner une couleur. On peut aussi aller plus loin, jouer avec le larsen, jusqu'à ne plus avoir besoin de la guitare.

Il faut chercher ses limites, et moi j'aime bien garder la guitare au centre de tout. 
Aussi, avec les ordinateurs, cela devient un autre instrument. J'aime bien avoir à ma disposition quand je joue des sons que je ne peux pas faire avec la guitare, des sons enregistrés, plus électroniques. J'utilise mon Iphone - les applications et l'ipod - pour les enclencher, je n'ai pas trouvé de solution satisfaisante avec un ordinateur. Je vois les possibilités de ce rêve sonore, mais il y a toutes les questions d'incompatibilité entre les sons enregistrés et l'ampli de la guitare, ou de la guitare avec une sonorisation plus « hi-fi ». Là il y a tout un parcours, je ne sais pas ce que ça pourrait devenir. Mais je n'ai pas envie de prendre le pas d'avoir un ordinateur sur scène.

Pourquoi pas (d'ordinateur sur scène)?

Hasse Poulsen: C'est une question de son, il ne me plaît pas. Il y a un son «macintosh » reconnaissable comme le son « stratocaster ». Je ne me sens pas attiré par les ordinateurs, déjà on passe tellement du temps devant ces machins que ça sera insupportable de les utiliser pour faire de la musique aussi. Je suis très attiré par des choses primitives, des choses qui ne marchent pas, des fautes, les hasards. Sur l'ordinateur il y a beaucoup de préparation, c'est assez - ou trop - parfait.

Les pédales - effets : est-ce que pour toi ces choses qui sont «autour » de la guitare font partie intégrante de ton instrument?

Hasse Poulsen : Oui, absolument. J'ai travaillé pendant cinq-six ans avec Louis Sclavis dans l'orchestre Napoli's Walls, et on avait toujours le même sonorisateur, Gilles Olivesi. J'ai beaucoup appris avec lui sur comment mixer et comment placer le son de la guitare. J'ai vu qu'il utilisait toujours certains types de délais, de compressions et d'équaliseurs. J'utilise un pédalier TC Electronics pour les effets. Je manipule aussi beaucoup les saturations, pendant longtemps j'ai été insatisfait avec les saturations, mais maintenant j'ai trouvé. En fait, avec ce genre de machines et d'effets, il faut comprendre comment fonctionne l'effet pour avoir un bon résultat. Par exemple, pour une bonne distorsion, il faut recycler le son deux fois à travers l'effet, et aussi utiliser un noise gate assez sévère pour couper les bruits parasites de la guitare acoustique.

Acoustique - électrique

Hasse Poulsen : Il y a trente ans il aurait été difficile de faire ce mélange acoustique électrique. Les microphones se sont beaucoup améliorés depuis. Les esthétiques ont beaucoup changé aussi. À l'époque l'on cherchait des sons qui ressembleraient aux vrais sons acoustiques. Maintenant avec la production de disques et les idées de production, ce n'est plus tellement un but en soi.

Longtemps j'ai utilisé un système de capteur acoustique Fishman, mais plus récemment j'ai installé un micro de guitare électrique de type Danelectro sur la rosace de ma Guild. Il est puissant, il marche très bien avec les effets et les saturations. J'aime bien parfois avoir un son un peu sale à la Keith Richards. Et surtout je trouve le mélange des deux génial: un microphone devant la guitare acoustique et un autre devant l'ampli donne un son vraiment spécial. L'amplification et le travail de studio deviennent une partie de notre son.

Conscience du son

Hasse Poulsen : Dans ce cadre, le son « naturel » ne fait plus sens, cela n'existe pas. Le type de microphone, son placement, le préampli, traitement, câble... ont tous une incidence sur le son. Il n'y a rien de naturel, et je pense que cette idée est devenue 
habituelle maintenant. L'on produit des sons qu'on a envie et besoin d'entendre pour le moment.

Il faut développer une conscience et une précision du son. Les gens sont souvent impressionnés par le son de groupe de notre trio Das Kapital. Cela vient de notre conscience des fréquences : on est constamment en train de régler le son, de changer de mode de jeu, de s'adapter les uns aux autres en fonction du son d'ensemble.

C'est intéressant de voir qu'après tout le travail qu'on peut faire avec les préparations et les machines, on peut revenir à la guitare acoustique avec cette même conscience du sonore. Tout d'un coup, il y a une richesse de timbres et de placements qu'on n'aurait pas découverte autrement.

Le geste

Hasse Poulsen : Je me retrouve dans la tradition scandinave très fonctionnaliste. J'aime bien le geste intégré au jeu, j'ai vraiment du mal avec les grimaces, avec les mouvements parasites. Aussi, dans le classique, il peut y avoir des gestes illustratifs. Comme Glenn Gould qui se « dirige » d'une main pendant que l'autre joue. Je trouve cela prétentieux et insupportable. Une sorte d'illustration de ce qui se passe. Le geste est dans le physique, le son vient de la matière. Tu veux faire cette note ? FAIS cette note! Il ne s'agit pas d'illustrer que là on va jouer fort, mais de jouer fort tout court. Je trouve très beaux ces mouvements imprévisibles du corps qui essaient de se placer dans la difficulté et l'excitation de la musique, et cela donne des drôles de mouvements.

\section{Résistance matérielle}

Hasse Poulsen: J'essaie tout le temps de trouver quelque chose de pas évident à faire. Là il y a une résistance, cela permet de se renouveler. On peut essayer de jouer un country-western sans tempo, ou un ragtime atonal. Comment est-ce qu'on fait ce genre de choses? On peut y arriver, on peut le travailler, c'est dans le domaine des paradoxes.

J'ai un instrument assez difficile à jouer, une guitare lourde, avec de grosses cordes. J'essaie de jouer des choses pas évidentes avec cette guitare et il y a tout le temps une résistance physique. C'est dur de jouer des notes qui tiennent, de jouer vite. Il faut travailler avec ça, rentrer dans le bois, entraîner le corps. Ces difficultés font que la musique sonne, que ça vit.

\section{Contourner les références}

Hasse Poulsen : À une époque, pendant 5 à 6 semaines, j'ai remplacé un ami dans un lycée. Alors j'ai fait l'expérience de jouer des musiques pour les élèves. J’ai joué la $1^{\text {re }}$ partie de la sonate en La majeur de Mozart que j'aime beaucoup, c'est très beau... Cela ne les intéressait pas du tout!

J'ai joué du jazz aussi, Sonny Rollins, un de ses chefs-d'œuvre des années 1950. Ça ne les intéressait pas non plus! C'était " papa », «papy »... vieux ! Puis j'ai joué du rock, quelque chose de pas bien du tout, une démo d'un de leurs copains, vraiment rock comme n'importe qui en faisait il y a 15 ans. Et là ils ont trouvé ça cool! C'était très stéréotypé.

Après j'ai joué Lux Aeterna de Ligeti, pièce assez radicale pour chœur et bois, qui figure dans l'odyssée de l'espace de Stanley Kubrick. Et là les réactions étaient fortes, quelqu'un disait «c'est comme être allongé dans de l'herbe un jour d'été, je me sens 
très léger. " L'autre fille disait "c'est comme être enfermé dans une cave, c'est tout humide. " Les réactions étaient très contrastées, et j'ai compris quelque chose, il y avait une musique sur laquelle ils ne pouvaient pas mettre de mots précis. Ils étaient obligés de puiser dans leurs émotions ! Ça me donnait un argument pour moi-même !

Pourquoi on fait de la nouvelle musique? Je pense que c'est important pour toute forme d'art de savoir pourquoi on fait quelque chose de nouveau, pourquoi on ne répète pas ce qui a prouvé sa valeur, en le raffinant, en le perfectionnant.

Je pense que pour parler aux émotions, il faut faire quelque chose qui est au-delà du langage. Il faut aller autour, contourner les mots, parce que les mots sont des barrières, l'art doit parler aux émotions.

Donc il faut contourner l'idée qu'on peut se faire de manière immédiate avec le mot?

Hasse Poulsen : Je trouve que dès qu'on nomme quelque chose, on n'est plus obligé de le regarder vraiment. Avec les mots on pense connaître la chose dont on parle, mais en réalité il n'en est rien.

Tu valorises l'idée de la nouveauté?

Hasse Poulsen : Quand on contourne ce qu'on peut exprimer en mots, on est obligé de réagir, de sentir. Ça nous réveille, ça nous fait nous sentir vivant. Je pense que l'idée de l'art c'est de nous mettre en rapport avec la vie.

Est-ce que l'artiste doit aller devant les mots, courir très vite pour ne pas se faire rattraper par les mots?

Hasse Poulsen : Bien sûr, c'est la leçon qu'on peut voir partout.

Imperfection

Hasse Poulsen: La musique que je fais, que j'appelle le jazz, est une musique imparfaite, bâtarde. Ce n'est pas comme de la musique symphonique, ce n'est jamais parfait. Il y a tout le temps des imperfections, des tensions, qui constituent l'ensemble. C'est cela qui donne l'énergie, l'espace.

Une image fondamentale pour moi c'est quand j'étais tout petit, j'habitais au Kenya et je parlais un peu Swahili. Un jour je devais faire la traduction pour mes parents qui voulaient acheter une statuette à un artisan local. Il ne voulait pas vendre la statue parce qu'il ne l'avait pas fini. Il fallait lui expliquer - via ce garçon de 6 ans que j'étais - que ma mère préférait cette statuette pas finie, polie, lisse, que son aspect rugueux lui plaisait d'avantage que les statuettes finies.

Pour moi cette statuette est un mythe fondateur. À l'époque, je ne pouvais pas comprendre pourquoi ma mère ne voulait pas acheter une des statuettes finies.

Il faut s'arrêter avant que la chose ne soit parfaite, avant que les choses deviennent trop polies, lisses. On peut travailler très consciemment là-dessus. 


\section{AUTEUR}

OTSO LÄHDEOJA

CICM, université Paris 8, MSH Paris Nord 